\title{
Pathologically confirmed brain metastases from primary uterine cervical tumors: two cases and a literature review
}

\author{
Yalan $\mathrm{Bi}^{1}$ and Lei $\mathrm{Li}^{2^{*}}$ (I)
}

\begin{abstract}
Background: Pathologically confirmed brain metastasis from primary cervical cancer is extremely rare. Herein, we report two cases of intracranial metastasis from cervical cancer that were histopathologically confirmed after surgical excision. In addition, we conducted a literature review to characterize the clinical manifestation, pathogenesis, and treatment of these patients.
\end{abstract}

Case summary: Among the 1800 patients with primary cervical cancer who received therapy at our center from 2010 to 2018, two patients (0.1\%) had definite histopathological evidence of brain metastasis. A 46-year-old female who had a history of poorly differentiated stage IIB cervical cancer with neuroendocrine differentiation presented with a solitary mass in the right occipital lobe 26 months after the initial diagnosis. She underwent surgery and chemotherapy but died of disease progression 9 months later. Another 55-year-old female diagnosed with poorly differentiated stage IVB cervical squamous cancer presented with a solitary mass in the right frontal lobe 16 months after simple hysterectomy. Twelve months later, multiple lesions were observed in the bilateral frontal-parietal lobe. The lesions were treated by surgery and stereotactic radiosurgery. The patient died of multiple organ failure 14 months later.

Conclusion: The pathogenesis and best management of brain metastasis from cervical cancer are not clear. Highly invasive subtypes or advanced cancer stages may be the key clinicopathological factors of brain metastasis. Surgical treatment is warranted in patients with a good health status and without metastasis to other sites.

Keywords: Cervical cancer, Brain metastasis, Pathogenesis, Chemotherapy, Radiotherapy

\section{Introduction}

Cervical cancer (CC) is the fourth most frequently diagnosed cancer and the fourth leading cause of cancerrelated death in females, with an estimated 570,000 cases and 311,000 deaths worldwide in 2018 [1]. While a typical treatment consisting of a combination of surgery, radiotherapy, and chemotherapy has been established for early-stage or locally advanced $\mathrm{CC}$, no standard treatment for metastatic disease has yet been established [2]. The 5-year survival rate in localized CC is $91.5 \%$; however, it is merely $16.5 \%$ in cases of metastasis [3]. CC mainly spreads locally to the pelvic and para-aortic lymph nodes via the lymphatic system [4]. However, CC

\footnotetext{
* Correspondence: lileigh@163.com

${ }^{2}$ Department of Obstetrics and Gynecology, Peking Union Medical College Hospital, Peking Union Medical College \& Chinese Academy of Medical Science, Shuaifuyuan No. 1, Dongcheng District, Beijing 100730, China Full list of author information is available at the end of the article
}

can also metastasize via the hematogenous pathway to more distant organs, with common sites being the lungs, liver, and bones [5]. Brain metastasis from primary CC is very rare, occurring in 0.4 to $2.3 \%$ of all patients [6]. To date, only approximately 140 cases of brain metastasis from CC have been reported [7]. Even fewer cases have been pathologically confirmed. Notably, an increase in brain metastasis has recently been observed [8]. Due to its rarity, no prospective clinical trials have been conducted to investigate optimal treatment strategies and prognostic factors, and poor overall survival-estimated to range from 2 to 8 months after the diagnosis of brain metastasis-has been reported [9].

In this report, we describe two cases of primary CC metastasis to the brain and conducted a literature review to investigate the clinical presentation, treatment, and prognosis of these patients. 


\section{Case presentation}

From January 2010 to December 2018, a total of 1800 patients with primary CC received therapy in our center, and recurrence occurred in 140 patients. After reviewing the medical cases, four patients $(0.2 \%)$ had suspected brain metastasis, and metastasis was confirmed in two patients $(0.1 \%)$ by pathological evaluation.

\section{Case 1}

A 46-year-old woman (gravidity 1, parity 1 ) was referred to the study center in May 2015 after she complained of irregular vaginal bleeding for 2 years. A pelvic examination revealed a $5-\mathrm{cm}$ cervical tumor involving the upper third of the vagina and infiltrating the distal compartment of the parametrium. A pelvic magnetic resonance imaging (MRI) scan revealed a $5.3 \times 4.8 \times 4.0 \mathrm{~cm}$ pelvic mass. Computed tomography (CT) scans of the chest and abdomen were negative for metastatic disease. Cervical biopsy confirmed poorly differentiated cervical carcinoma graded as International Federation of Gynecology and Obstetrics (FIGO) stage IIB. Immunohistochemical staining was positive for CK7, CgA, ER, PR, and PAX-8 expression but negative for CEA, Napsin A, P16, P40, P63, Syn, TTF-1, and CD56 (NK1) expression. The Ki-67 index was 70\%. Primary treatment consisted of concurrent chemoradiotherapy: external radiation to the pelvis $(60.0 \mathrm{~Gy} / 28 \mathrm{f})$ followed by intracavitary brachytherapy $(30 \mathrm{~Gy} / 5 \mathrm{f})$ and concurrent intravenous cisplatin $\left(40 \mathrm{mg} / \mathrm{m}^{2} /\right.$ week $)$ for 5 weeks. Complete response was achieved, as determined by imaging evaluation.

After a 1-year remission, in September 2016, the patient presented with a cough that had lasted several weeks. CT scan of the lungs showed multiple metastatic nodules, and the biopsy revealed a poorly differentiated carcinoma. She then received 6 cycles of chemotherapy (docetaxel and cisplatin for 3 cycles, 3 weeks per cycle, and docetaxel and oxaliplatin for another 3 cycles, 3 weeks per cycle, due to decreased renal perfusion). Three months later, the patient was admitted for neurological evaluation because of severe headache, projectile vomiting, and a left homotropic hemianopia. A subsequent brain MRI showed a solitary $3 \times 4 \times 5 \mathrm{~cm}$ heterogeneous cystic mass in the right occipital lobe with surrounding edema (Fig. 1a-d). T1-weighted imaging (T1WI) and T2WI showed a solid component of equal signal, while diffusion-weighted imaging (DWI) showed hyperintensity with decreased apparent diffusion coefficient (ADC) values. The mass was enhanced with contrast enhancement. The patient underwent surgical resection of the metastatic tumor in the right occipital lobe with neuronavigation on July 31, 2017. Her preoperative Karnofsky performance score (KPS) was 80. Histopathological examination showed a metastatic, poorly differentiated adenocarcinoma with neuroendocrine differentiation (Fig. 2a). Immunohistochemical
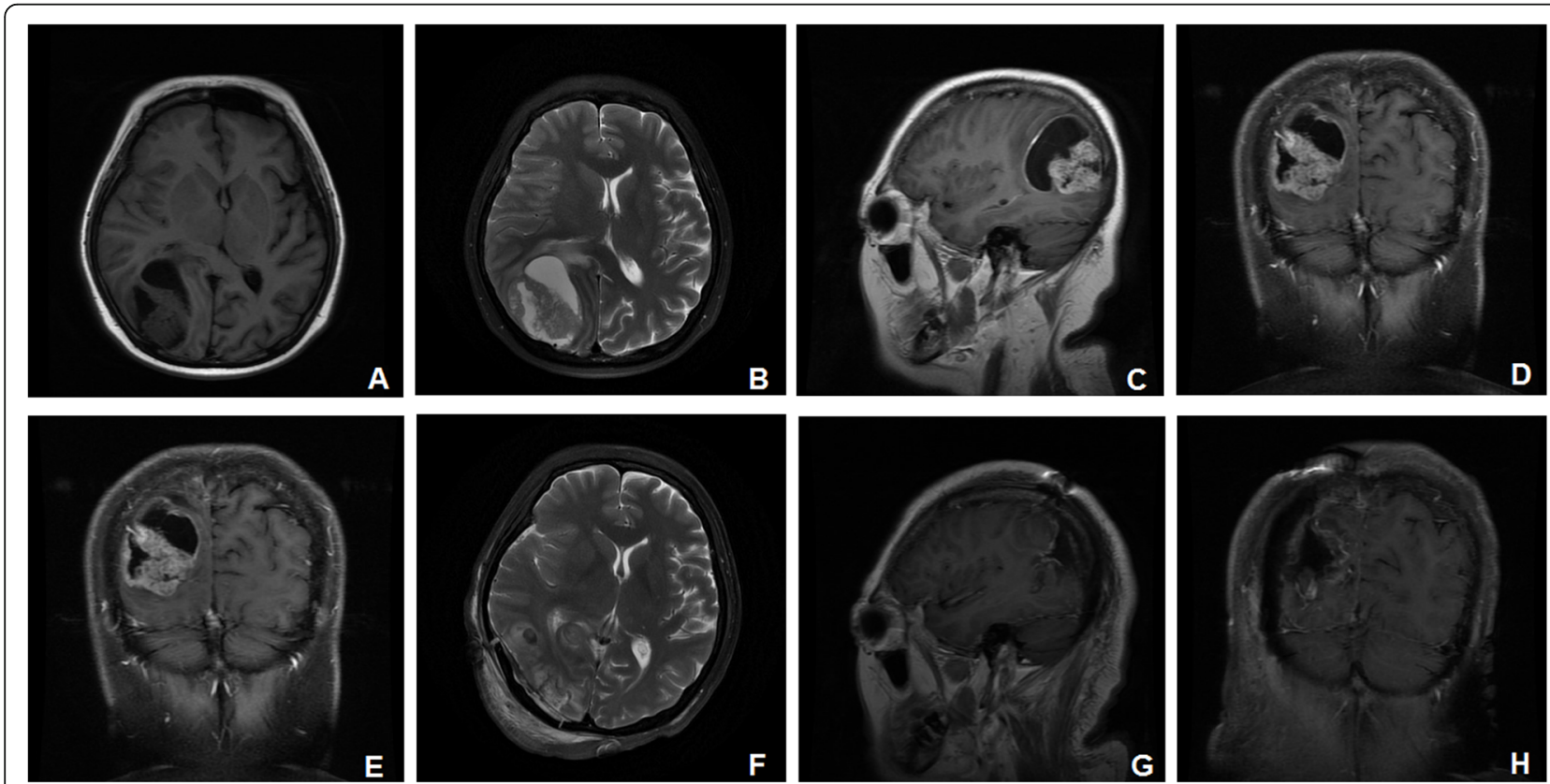

Fig. 1 A preoperative MRI scan of the brain on July 24, 2017, showed a solitary mass in the right occipital lobe (a-d). An MRI scan of the brain 1 day after the operation showed postoperative changes in the right occipital area, with gross total resection of the lesion on August 1 , 2017 (eh). The MRI sequences consisted of the following: T1-weighted imaging (T1WI) for $\mathbf{a}$, $\mathbf{g}$, and $\mathbf{h}$; T2WI for $\mathbf{b}$ and $\mathbf{f}$; and diffusion-weighted imaging (DWI) for $\mathbf{c}, \mathbf{d}$, and $\mathbf{e}$ 


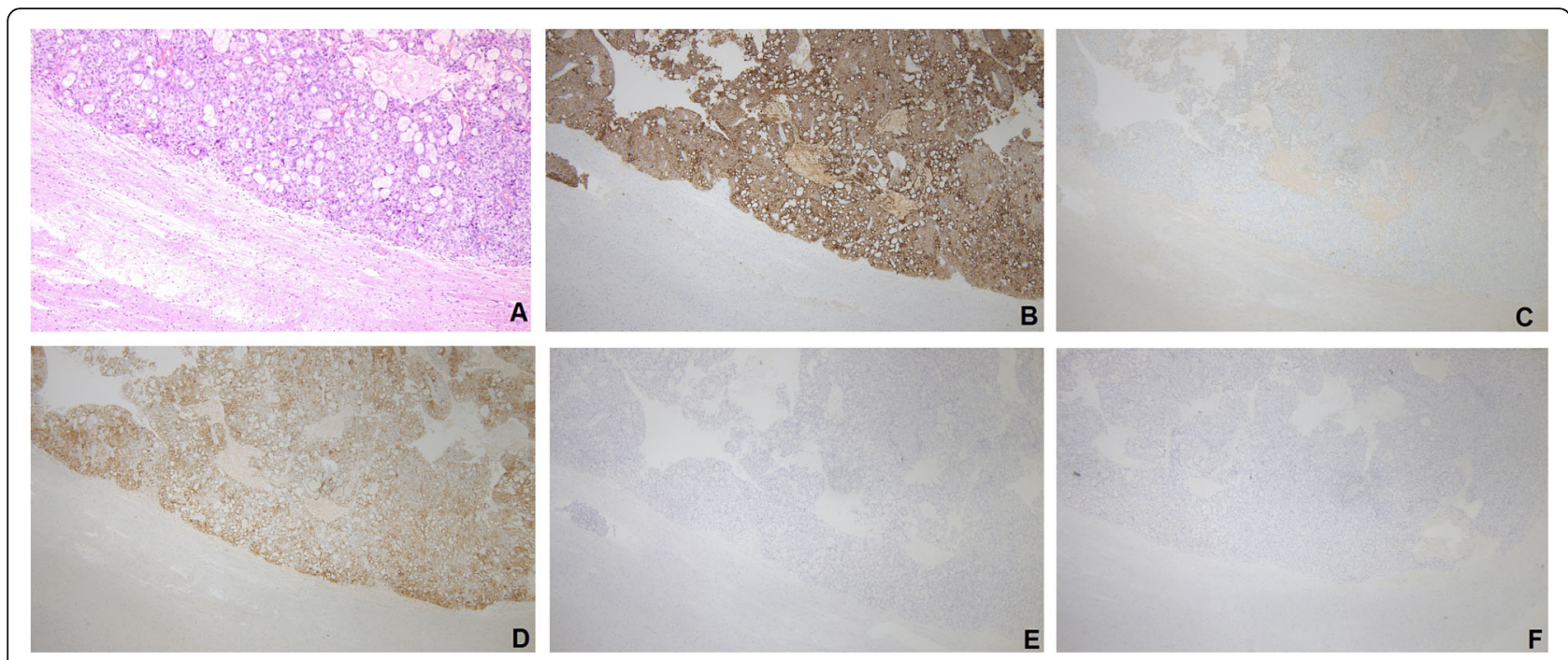

Fig. 2 Pathology of a metastatic brain tumor from CC, which shows poorly differentiated adenocarcinoma with neuroendocrine differentiation. a Hematoxylin and eosin staining, $\times 20$. Immunohistochemical staining showed strong, positive signals for CK7 (b), CK20 (c), P16 (d), P40 (e), and $\pi \mathrm{TT}-1(\mathbf{f}), \times 20$

staining showed strong positivity for CK7 and positivity for CK20, P16, P40, and TTF-1 (Fig. 2b-f). The patient experienced no postoperative complications and showed good recovery at the time of discharge. A postoperative brain MRI performed on August 1, 2017, showed gross total resection of the lesion (Fig. 1e-h). Only sheet signals of long/short T1 and T2 signals were observed, and DWI still showed hyperintensity with decreased ADC values. She refused radiotherapy for rapid progression in the liver and lungs and subsequently received 5 cycles of chemotherapy (liposomal doxorubicin and carboplatin, 4 weeks per cycle) until February 2018; she died of extensive metastasis to multiple organs 2 months later in April 2018, with an overall survival (OS) of 9 months after the brain surgery.

\section{Case 2}

A 55-year-old female underwent simple hysterectomy for assumed cervical intraepithelial neoplasia 3 (CIN 3) in July 2015 . However, pathology revealed poorly differentiated squamous cell cancer of the cervix with parametrial and full-thickness stromal involvement. Immunohistochemical staining was not performed in this case. Postoperative positron emission tomography (PET) revealed multiple metastatic sites, including the lungs, mediastinum, bones, and pelvic and supraclavicular lymph nodes, confirming a diagnosis of stage IVB cancer. She was transferred to our center and was treated by external radiation to the pelvis $(56.0 \mathrm{~Gy} / 28 \mathrm{f})$ followed by intracavitary brachytherapy (24Gy/4 f) and chemotherapy (cisplatin and fluorouracil, 3 weeks per cycle for
2 cycles, cisplatin and paclitaxel, 3 weeks per cycle for 6 cycles, and carboplatin and paclitaxel, 3 weeks per cycle for 1 cycle). Complete response was achieved.

In November 2016, she presented with a mild, intermittent headache, and an MRI scan of her brain showed a solitary mass in her right frontal lobe, which could not be resected due to the location of the tumor (Fig. 3a-c). Long T1 and long T2 signals with limited DWI diffusion were observed on MRI. The mass was enhanced with contrast enhancement. Consultation with a radiotherapist led to the recommendation of whole-brain radiation therapy (WBRT) for brain metastasis. The patient refused radiotherapy for fear of both potential complications and the misdiagnosis of metastasis. During the follow-up period, the patient had a mild headache, and MRI revealed a stable lesion. In November 2017, the patient presented with weakness and trembling in her right limbs. An MRI scan of her brain showed multiple heterogeneous solid masses in the bilateral frontal-parietal lobe with surrounding edema, suggestive of brain metastases. Short T1 and long T2 signals and slightly increased DWI signals were observed, which were all enhanced (Fig. 3d-f). A CT scan of her abdomen and pelvis did not reveal any new lesions. The patient underwent surgical resection of multiple metastases in the left frontal lobe with neuronavigation on January 30, 2018. Her preoperative KPS was 70. Histopathological examination of the resected tumor revealed metastatic squamous cell cancer from the cervix (Fig. 4). The lesion in her right frontal lobe was left in situ as the patient refused surgical treatment for fear of misdiagnosis because the lesion had remained stable over the past year, even 

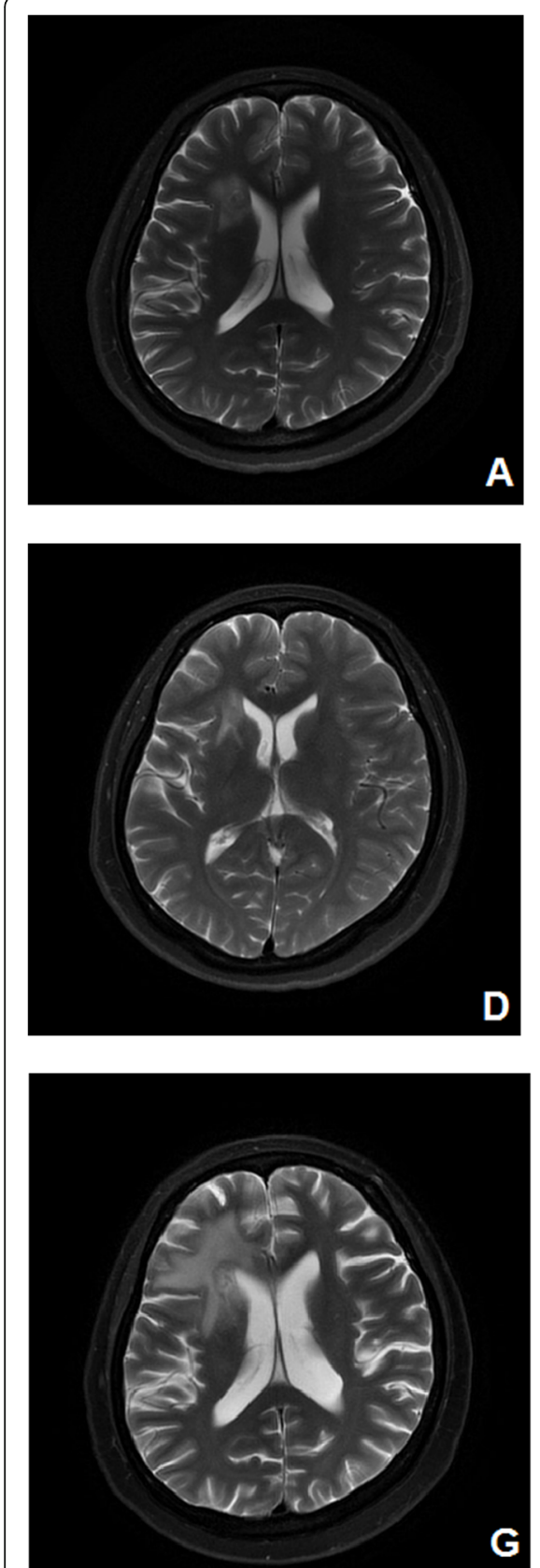
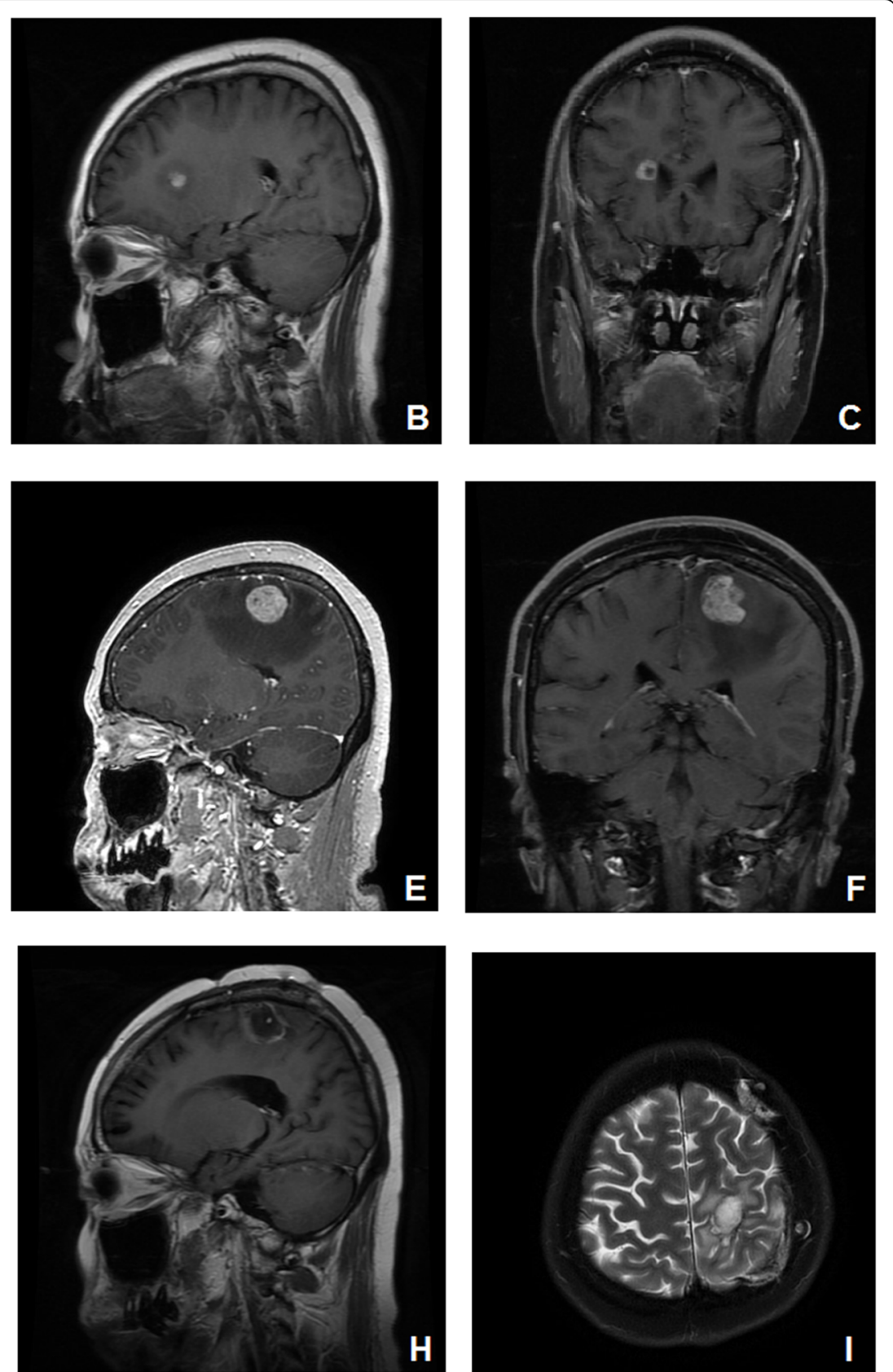

Fig. 3 A preoperative MRI scan of the brain on November 18, 2016, showed a solitary mass in the right frontal lobe (a-c). One year later on December 25, 2017, a preoperative MRI scan of the brain showed multiple lesions in the bilateral frontal-parietal lobe (d-f). Three months after the operation, an MRI scan of the brain performed on May 3, 2018, showed postoperative changes in the bilateral frontal-parietal lobe, with gross total resection of the lesions ( $\mathbf{g}-\mathbf{i})$. The MRI sequences consisted of the following: T2WI for $\mathbf{a}, \mathbf{d}, \mathbf{g}$, and $\mathbf{i}$; DWI for $\mathbf{b}, \mathbf{c}, \mathbf{e}$, and $\mathbf{f}$; and T1WI for $\mathbf{h}$

after close communication. Three months after the operation, an MRI scan of her brain showed gross total resection of the lesion, which showed long T1 and T2 signals and high DWI signals without decreased ADC values (Fig. $3 g-i$ ). The patient recovered well and showed no neurological complications postoperatively. She was subsequently treated with stereotactic radiosurgery (SRS) at a dose of $18 \mathrm{~Gy}$ in March 2018. She remained disease-free until December 2018 and died in March 2019 due to disease progression, with an OS of 14 months after the brain surgery.

\section{Discussion}

Brain metastasis from $\mathrm{CC}$ is rare. In a review of 1565 patients diagnosed with $\mathrm{CC}$, only 12 cases of brain metastasis $(0.76 \%)$ were identified [10]. Pathologically 

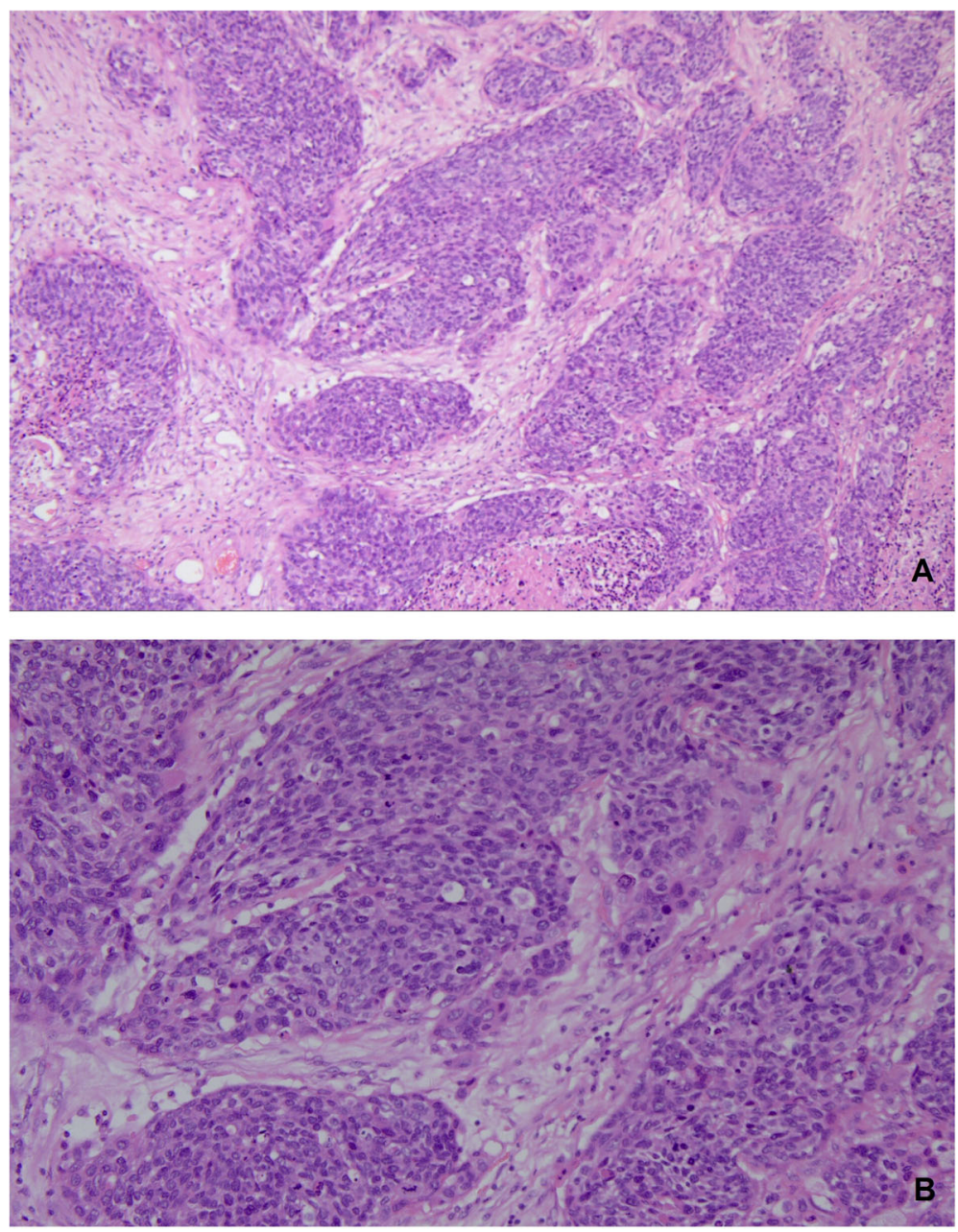

Fig. 4 Pathology of a metastatic brain tumor from cervical squamous carcinoma (hematoxylin and eosin staining, $\times 20$ in $\mathbf{a}$ and $\times 50$ in b)

confirmed brain metastasis is even rarer. Among the 149 patients with CC metastasis to the brain reported in the English literature, only 46 patients (30.9\%) underwent surgical excision and had corresponding histopathological evidence [7]. These findings are contrary to those for other malignancies. Metastatic brain tumors are the most common intracranial neoplasms in adults, with an incidence of $10-15 \%$ of all patients with systemic malignancies [11, 12]. Recently, an increase in the incidence of brain metastasis has been noted, largely due to earlier detection as a result of improved imaging methods [13]. The most common primary cancers that metastasize to the brain are lung cancer (45\%), breast cancer (15\%), melanoma (10\%), and colorectal cancer $(5 \%)[14,15]$.

These patients with brain metastasis from CC had a distinct natural history and obvious neurological symptoms. The median age of the onset of brain metastasis was 48 years, ranging from 29 to 87 years $[7,16,17]$. While some patients had brain metastasis at the time of primary cancer diagnosis $[18,19]$, the median interval between primary cancer diagnosis and brain metastasis was 17.2 months [7], and the longest interval reported was 127.2 months [9]. The intervals in our cases from the time of $\mathrm{CC}$ diagnosis to brain metastasis were 26 and 16 months. Patients suffering from brain metastasis can present with various symptoms, including headaches, seizures, cognitive impairment, fatigue, and focal deficits [20]. The most common symptoms were headache $(31 \%)$, hemiplegia $(16 \%)$, seizure $(11 \%)$, and confusion (9\%) [7]. Most patients had multiple lesions (55\%), and the most common area of metastatic lesions was the supratentorial region (75\%) [7].

The pathogenic mechanisms underlying brain metastasis from CC remain unclear. It has been reported that the interaction between tumor cells and brain cells within the brain microenvironment results in the release of various cytokines that subsequently promote tumor growth [21-24]. Another significant bottleneck in understanding the pathogenesis of brain metastasis is the limited availability of nutrients in the brain. Metastatic 
brain tissue is able to utilize substrates from glycolysis, the pentose phosphate pathway [25], and the glutamine pathway, including pyrimidines, purines, and nonessential amino acids, as both energy sources and cellular building blocks [26]. However, these findings were almost all discovered in cases of other malignancies, not in cases of CC. No substantial evidence has been uncovered to reveal the pathogenesis of brain metastasis from CC.

Among various clinicopathological factors, highly invasive subtypes or advanced stages of CC may be the key factors of metastasis to the brain. Most brain metastases from CC have been reported to be poorly differentiated [27], and nonsquamous subtypes account for $32 \%$ or more cases of brain metastasis $[7,28]$, which is significantly higher than the proportion in primary cervical lesions. It is worth noting that almost half of the patients (up to $40 \%$ ) with intracranial metastasis from CC had advanced stage disease $[7,16]$. In another report of 27 patients, $21(77.8 \%)$ had stage IIB disease or more advanced stages of disease [29]. Most patients with brain metastasis also developed recurrence at extracranial sites, including in the lungs (39\%), bones (16\%), and abdomen/pelvis (16\%) [7]. Small cell neuroendocrine carcinoma of the cervix is prone to brain metastasis and has an unfavorable prognosis [30]. Several reports have also documented brain metastasis in cervical neuroendocrine tumors $[28,31]$. In conclusion, an advanced stage and nonsquamous subtype contribute significantly to brain metastasis, which is similar to the risk factors for bone metastasis $[32,33]$ or metastasis to other distant sites beyond the pelvic cavity [34]. In our two cases, the first patient had poorly differentiated stage IIB cervical carcinoma with neuroendocrine differentiation and the second patient had poorly differentiated stage IVB CC. However, more cases are needed to further uncover the high risk factors of brain metastasis from CC.

There is still no consensus on the most effective therapy for patients with brain metastasis. WBRT was suggested in the 1980s to prevent neurological death by reducing the tumor volume and treating micrometastases. WBRT is also an option for patients with uncontrolled primary disease or extensive systemic metastases; it is the treatment of choice in patients who are not suitable for surgery or SRS [35] and is used an adjuvant treatment to surgery or SRS to increase local and distant tumor control [36]. SRS employs multiple, highly focused, convergent beams to deliver a high dose of radiation to intracranial targets [37], which allows radiation to be delivered with a steep radiation dose drop-off outside the targeted tumor border, minimizing the risk of damaging the surrounding normal brain tissue [38]. SRS may be preferred to WBRT in select patients who have undergone the total resection of one to three metastatic brain lesions, as in our report of case 2. In recent studies, WBRT was associated with various short-term and long-term radiation-induced injuries to the brain. Adding stereotactic radiosurgery to WBRT provides better local control than WBRT alone, as shown in a previously reported review and meta-analysis [39]. Brown et al. [40] studied 194 patients who underwent brain metastasis resection and found no difference in survival between SRS and WBRT, while cognitive impairment was more frequent in patients who received WBRT than in those who received SRS at 6 months $(85 \%$ vs. $52 \%$ of patients, $p<0.001)$. Novel therapies for brain metastasis from malignancies are emerging, including targeted therapy. These reports include cases of melanoma brain metastasis treated with ipilimumab $[41,42]$ and cases of brain metastasis from melanoma and non-small-cell lung cancer treated with pembrolizumab [43]. To the best of our knowledge, there are no targeted therapies for brain metastasis from CC.

Surgical resection is now considered for patients with a radioresistant primary histological type, those with a large tumor volume causing brain shift, those with symptoms refractory to medical treatment, and those with controlled disease at the primary site without systemic metastasis [13]. However, very little of this experience is in the field of CC. A small cohort study revealed that patients who underwent surgery for brain metastasis exhibited better survival than patients receiving only WBRT [44]. Favorable prognostic factors for prolonged survival after the surgical resection of central nervous system metastases are a good patient performance status, a long disease-free interval, an absence of other systemic diseases, and resectability, preferably with clear margins [45]. Additionally, resection allows for the histological confirmation of metastasis and differentiation with necrosis [46, 47]. It is alarming that this evidence was not all drawn from the treatment of $\mathrm{CC}$ but was instead drawn from the treatment of heterogeneous tumor types. Thus, the adoption and generalization of these conclusions in CC patients should be considered with caution. The surgical timing for brain metastasis has not been explicitly clarified. Surgical treatment in our two cases achieved transient disease-free periods of 7 and 11 months, demonstrating the positive role of surgery. Interestingly, in case 2, a single right frontal lobe lesion was stable for 1 year before new lesions appeared in the bilateral frontal-parietal lobe.

The survival of brain metastasis from CC is very poor; the mean and median survival times after the diagnosis of brain metastasis were reported to be 7 and 4.6 months, respectively, in a literature review [7]. Records from 81 patients with uterine cancer metastasis to the brain in Japan showed a median OS of 7 months (95\% CI 4-10) [48]. In another study, the mean survival was 8.2 months after central nervous system metastasis was 
discovered [29]. It has been suggested that the treatment modality, particularly combined therapies, is significantly related to OS [49]. The poor prognosis is probably due to metastasis to multiple sites rather than to the brain alone. In our cases, after brain surgery and multimodal therapy, the OS in cases 1 and 2 was 9 and 14 months, respectively. Both patients died of disease progression, even after successful management of the brain loci.

\section{Conclusion}

Pathologically confirmed brain metastasis from CC is rare. Although management varies based on individual characteristics, surgery appears to be critical for both disease control and pathological confirmation. Highly invasive subtypes or advanced stages of $\mathrm{CC}$ may be the key factors of brain metastasis. Future large-scale reports are needed to clarify the pathogenesis of and optimal treatment approach for brain metastasis from CC.

\section{Acknowledgements}

None.

\section{Statement of submission}

The paper is not under consideration by another journal, and the results presented in this work have not been presented or published previously.

\section{Authors' contributions}

LL conceived of the original idea for the study, interpreted the results, carried out the statistical analysis, edited the paper, and was an overall guarantor. LL obtained the ethical approval, contributed to the preparation of the dataset, interpreted the results, and contributed to drafts of the paper. $L L$ and $Y B$ contributed to the study design and interpretation of the results and commented on drafts of the paper. YB was responsible for the interpretation of the pathological evaluations. Both authors read and approved the final manuscript.

\section{Funding}

This study was supported by the Chinese Academy of Medical Sciences Initiative for Innovative Medicine (CAMS-2017-I2M-1-002) and by the National Science-Technology Support Plan Projects (2015BAl13B04). The funders played no role in the study design, data collection and analysis, decision to publish, or preparation of the manuscript.

\section{Availability of data and materials}

All the data in this report have been presented in the manuscript.

\section{Ethics approval and consent to participate}

The Institutional Review Board of Peking Union Medical College Hospital approved this study (No. ZS-1427). The registration number is NCT03291236 (clinicaltrials.gov).

\section{Consent for publication}

The patients in this report had both given consent for publication before their deaths, and all presentations in this report have permission to be published.

\section{Competing interests}

The authors declare that they have no competing interests.

\section{Author details}

'Department of Pathology, Peking Union Medical College Hospital, Peking Union Medical College \& Chinese Academy of Medical Science, Beijing 100730, China. ${ }^{2}$ Department of Obstetrics and Gynecology, Peking Union Medical College Hospital, Peking Union Medical College \& Chinese Academy of Medical Science, Shuaifuyuan No. 1, Dongcheng District, Beijing 100730, China.
Received: 9 July 2019 Accepted: 3 October 2019

Published online: 31 October 2019

\section{References}

1. Bray F, Ferlay J, Soerjomataram I, et al. Global cancer statistics 2018: GLOBOCAN estimates of incidence and mortality worldwide for 36 cancers in 185 countries. CA Cancer J Clin. 2018:68(6):394-424.

2. van Meir H, Kenter GG, Burggraaf J, et al. The need for improvement of the treatment of advanced and metastatic cervical cancer, the rationale for combined chemo-immunotherapy. Anti Cancer Agents Med Chem. 2014; 14(2):190-203.

3. Li H, Wu X, Cheng X. Advances in diagnosis and treatment of metastatic cervical cancer. J Gynecol Oncol. 2016;27(4):e43.

4. Agrawal A, Kumar A, Sinha AK, et al. Intracranial metastases from carcinoma of the cervix. Singap Med J. 2007:48(5):e154-6.

5. Amita M, Sudeep G, Rekha W, et al. Brain metastasis from cervical carcinoma--a case report. MedGenMed. 2005;7(1):26.

6. Divine LM, Kizer NT, Hagemann AR, et al. Clinicopathologic characteristics and survival of patients with gynecologic malignancies metastatic to the brain. Gynecol Oncol. 2016;142(1):76-82.

7. Fetcko K, Gondim DD, Bonnin JM, et al. Cervical cancer metastasis to the brain: a case report and review of literature. Surg Neurol Int. 2017:8:181.

8. Peters $\mathrm{P}$, Bandi H, Efendy J, et al. Rapid growth of cervical cancer metastasis in the brain. J Clin Neurosci. 2010;17(9):1211-2.

9. Chung SB, Jo Kl, Seol HJ, et al. Radiosurgery to palliate symptoms in brain metastases from uterine cervix cancer. Acta Neurochir. 2013:155(3):399-405.

10. Chura JC, Shukla K, Argenta PA. Brain metastasis from cervical carcinoma. Int J Gynecol Cancer. 2007;17(1):141-6.

11. Schoggl A, Kitz K, Reddy M, et al. Defining the role of stereotactic radiosurgery versus microsurgery in the treatment of single brain metastases. Acta Neurochir. 2000;142(6):621-6.

12. Al-Shamy G, Sawaya R. Management of brain metastases: the indispensable role of surgery. J Neuro-Oncol. 2009;92(3):275-82.

13. Hatiboglu MA, Akdur K, Sawaya R. Neurosurgical management of patients with brain metastasis. Neurosurg Rev. 2018. https://doi.org/10.1007/s10143018-1013-6. [Epub ahead of print].

14. Nayak L, Lee EQ, Wen PY. Epidemiology of brain metastases. Curr Oncol Rep. 2012;14(1):48-54.

15. Patchell RA. The management of brain metastases. Cancer Treat Rev. 2003; 29(6):533-40.

16. Lefkowitz $D$, Asconape J, Biller J. Intracranial metastases from carcinoma of the cervix. South Med J. 1983;76(4):519-21.

17. Nasu K, Satoh T, Nishio S, et al. Clinicopathologic features of brain metastases from gynecologic malignancies: a retrospective study of 139 cases (KCOG-G1001s trial). Gynecol Oncol. 2013;128(2):198-203.

18. Buchsbaum HJ, Rice AC. Cerebral metastasis in cervical carcinoma. Am J Obstet Gynecol. 1972;114(2):276-8.

19. Cordeiro JG, Prevedello DM, da Silva Ditzel LF, et al. Cerebral metastasis of cervical uterine cancer: report of three cases. Arq Neuropsiquiatr. 2006; 64(2A):300-2.

20. Noh T, Walbert T. Brain metastasis: clinical manifestations, symptom management, and palliative care. Handb Clin Neurol. 2018;149:75-88.

21. Raza M, Prasad P, Gupta P, et al. Perspectives on the role of brain cellular players in cancer-associated brain metastasis: translational approach to understand molecular mechanism of tumor progression. Cancer Metastasis Rev. 2018;37(4):791-804.

22. Bos PD, Zhang XH, Nadal C, et al. Genes that mediate breast cancer metastasis to the brain. Nature. 2009;459(7249):1005-9.

23. Valiente $M$, Obenauf $A C$, Jin $X$, et al. Serpins promote cancer cell survival and vascular co-option in brain metastasis. Cell. 2014;156(5):1002-16.

24. Wei J, Gabrusiewicz K, Heimberger A. The controversial role of microglia in malignant gliomas. Clin Dev Immunol. 2013;2013:285246.

25. Chen El, Hewel J, Krueger JS, et al. Adaptation of energy metabolism in breast cancer brain metastases. Cancer Res, 2007:67(4):1472-86.

26. Chen J, Lee HJ, Wu X, et al. Gain of glucose-independent growth upon metastasis of breast cancer cells to the brain. Cancer Res. 2015;75(3):554-65.

27. Mahmoud-Ahmed AS, Suh JH, Barnett GH, et al. Tumor distribution and survival in six patients with brain metastases from cervical carcinoma. Gynecol Oncol. 2001;81(2):196-200.

28. Hwang JH, Yoo HJ, Lim MC, et al. Brain metastasis in patients with uterine cervical cancer. J Obstet Gynaecol Res. 2013;39(1):287-91. 
29. Cacho-Diaz B, Lorenzana-Mendoza NA, Michel-Ortega RM, et al. Central nervous system metastases in patients with cervical carcinoma. Int J Gynecol Cancer. 2016;26(9):1686-9.

30. Lan-Fang L, Hai-Yan S, Zuo-Ming Y, et al. Small cell neuroendocrine carcinoma of the cervix: analysis of the prognosis and role of radiation therapy for 43 cases. Eur J Gynaecol Oncol. 2012;33(1):68-73.

31. Komiyama S, Nishio E, Torii Y, et al. A case of primary uterine cervical neuroendocrine tumor with meningeal carcinomatosis confirmed by diagnostic imaging and autopsy. Int J Clin Oncol. 2011;16(5):581-6.

32. Yoon $\mathrm{A}$, Choi $\mathrm{CH}, \mathrm{Kim} \mathrm{HJ}$, et al. Contributing factors for bone metastasis in uterine cervical cancer. Int J Gynecol Cancer. 2013;23(7):1311-7.

33. Zhang Y, Guo X, Wang G, et al. Real-world study of the incidence, risk factors, and prognostic factors associated with bone metastases in women with uterine cervical cancer using Surveillance, Epidemiology, and End Results (SEER) data analysis. Med Sci Monit. 2018:24:6387-97.

34. Kodama J, Seki N, Nakamura K, et al. Prognostic factors in pathologic parametrium-positive patients with stage IB-IIB cervical cancer treated by radical surgery and adjuvant therapy. Gynecol Oncol. 2007;105(3):757-61.

35. Hatiboglu MA, Tuzgen S, Akdur K, et al. Treatment of high numbers of brain metastases with gamma knife radiosurgery: a review. Acta Neurochir. 2016;158(4):625-34.

36. Churilla TM, Handorf E, Collette $\mathrm{S}$, et al. Whole brain radiotherapy after stereotactic radiosurgery or surgical resection among patients with one to three brain metastases and favorable prognoses: a secondary analysis of EORTC 22952-26001. Ann Oncol. 2017;28(10):2588-94.

37. Soffietti R, Ruda R, Trevisan E. Brain metastases: current management and new developments. Curr Opin Oncol. 2008;20(6):676-84.

38. Baumert BG, Rutten I, Dehing-Oberije C, et al. A pathology-based substrate for target definition in radiosurgery of brain metastases. Int J Radiat Oncol Biol Phys. 2006;66(1):187-94.

39. National Comprehensive Cancer Network (NCCN). Survivorship (version 2. 2014). nccn.org/professionals/physician_gls/pdf/survivorship.pdf. Accessed 14 Dec 2014.

40. Brown PD, Ballman KV, Cerhan JH, et al. Postoperative stereotactic radiosurgery compared with whole brain radiotherapy for resected metastatic brain disease (NCCTG N107C/CEC.3): a multicentre, randomised, controlled, phase 3 trial. Lancet Oncol. 2017;18(8):1049-60.

41. Queirolo P, Spagnolo F, Ascierto PA, et al. Efficacy and safety of ipilimumab in patients with advanced melanoma and brain metastases. J Neuro-Oncol. 2014;118(1):109-16.

42. Knisely JP, Yu JB, Flanigan J, et al. Radiosurgery for melanoma brain metastases in the ipilimumab era and the possibility of longer survival. J Neurosurg. 2012;117(2):227-33.

43. Goldberg SB, Gettinger SN, Mahajan A, et al. Pembrolizumab for patients with melanoma or non-small-cell lung cancer and untreated brain metastases: early analysis of a non-randomised, open-label, phase 2 trial. Lancet Oncol. 2016;17(7):976-83.

44. Vecht CJ, Haaxma-Reiche H, Noordijk EM, et al. Treatment of single brain metastasis: radiotherapy alone or combined with neurosurgery? Ann Neurol. 1993;33(6):583-90.

45. Tangjitgamol S, Levenback CF, Beller U, et al. Role of surgical resection for lung, liver, and central nervous system metastases in patients with gynecological cancer: a literature review. Int J Gynecol Cancer. 2004;14(3):399-422.

46. Wronski M, Arbit E, McCormick B. Surgical treatment of 70 patients with brain metastases from breast carcinoma. Cancer. 1997;80(9):1746-54

47. Patchell RA, Tibbs PA, Walsh JW, et al. A randomized trial of surgery in the treatment of single metastases to the brain. N Engl J Med. 1990:322(8):494-500

48. Hayashi N, Takahashi H, Hasegawa Y, et al. A nationwide multi-institutional retrospective study to identify prognostic factors and develop a graded prognostic assessment system for patients with brain metastases from uterine corpus and cervical cancer. BMC Cancer. 2017;17(1):397.

49. Kim YZ, Kwon JH, Lim S. A clinical analysis of brain metastasis in gynecologic cancer: a retrospective multi-institute analysis. J Korean Med Sci. 2015;30(1):66-73.

\section{Publisher's Note}

Springer Nature remains neutral with regard to jurisdictional claims in published maps and institutional affiliations.

\section{Ready to submit your research? Choose BMC and benefit from:}

- fast, convenient online submission

- thorough peer review by experienced researchers in your field

- rapid publication on acceptance

- support for research data, including large and complex data types

- gold Open Access which fosters wider collaboration and increased citations

- maximum visibility for your research: over $100 \mathrm{M}$ website views per year

At BMC, research is always in progress.

Learn more biomedcentral.com/submissions 